\title{
The Wear of Cutting Pellets Coated with TiAIN During Machining of a Cobalt Superalloy (FSX-414)
}

\author{
Sebhi Amar, Douib Nadir \\ Department of Mechanical Engineering, Faculty of Technology, University Med Boudiaf, M'Sila, Algeria \\ Email address: \\ Sebhiamar@yahoo.fr (S. Amar), Mouad-douib@yahoo.fr (D. Nadir)
}

To cite this article:

Sebhi Amar, Douib Nadir. The Wear of Cutting Pellets Coated with TiAlN During Machining of a Cobalt Superalloy (FSX-414). American Journal of Software Engineering and Applications. Vol. 5, No. 6, 2016, pp. 56-60. doi: 10.11648/j.ajsea.20160506.13

Received: December 6, 2016; Accepted: December 23, 2016; Published: January 23, 2017

\begin{abstract}
For the machining of superalloys, unconventional methods such as electroerosion, electrochemistry and others are frequently used due to the hardness of the material. Although, for some other operations, theconventional machining is essential. Because of their high hardness and improved mechanical properties, these superalloys require specific materials means such as machines and tools. To get an idea of the behavior of such a material in particular fields such as gas turbines, nuclear reactors, the industrialists have been interested for many years in the development and characterization of new materials to optimize the wear of the cutting tools and produce at a lower cost. In this context, the study of the tribological behavior of the cobalt-based superalloy FSX-414 standard 9001F in contact with the TiAlN-coated carbide inserts provides important information in the case of the turbine blade industry. The article discusses the productivity of gas turbine blades of the thermal power plant electricity production. The material to be machined is a superalloy which requires a carbide tool with adequate coating (TiN, TiC, TiAlN, TiCN...). This research eté preceded by an article about the couple material / tool (FSX414 / TiN coated carbide). A comparison of results has been made to optimize the cutting process and start production with lower cost. The lifetime of the cutting tool is mainly related to the development of two types of wear: flankwear of the sidewall and crater wear. This phenomenon of wear seems to be progressive, it develops during the cutting, which affects the quality of the machining, the productivity, and in particularly it may lead to the destruction of the tool in case of excessive wear. For the measurement of wear, an indirect method is used by coupling a Touptek photon camera to a microscope and capture the image to be processed by Toupview analysis software. It has been shown that the coating is the most influential parameter, followed by the sliding velocity. In this context, a focused research on the wear of TiAlN-coated Platelets in contact with this superalloy which is resistant to wear at high temperature has been developed.
\end{abstract}

Keywords: Cutting Speed, Flank Wear, Friction Wear Crater, Milling, Tribology

\section{Introduction}

This research comes after a work carried out in a previous step [1]. In order to compare the results and confirm the behavior of the friction coefficient obtained in the previous work [2], series of tests are necessary to confine these results.

Wear is a complex mechanism that is difficult to interpret, causing debris emission with loss of weight, size and shape transformation and behind it is accompanied by a physical and chemical surface transformation. Cobalt-based alloys have a better resistance to high temperature corrosion than nickel based alloys. They are more expensive and more difficult to machine because of their wear resistance.

Several surveillance methods of the wear of cutting tools have been applied to satisfy the productivity needs. The exploitation of different kinds of parameters during machining such as signals, cutting forces, acoustic emission and vibration is used to extract the information needed for effective monitoring using indirect techniques that describe the roughness of the workpiece, the cutting power and vibration generated during processing [3]. In the same way, other indirect methods in parallel monitor the development of the wear at the machining site. There is always a quite small scale on which they are measurable and their description does not date from yesterday [4, 5]. This tribological wear phenomena appears to be progressive, develops during cutting, which affects the quality of the machining, productivity and particularly, it may lead to the destruction of the tool after an excessive wear [6]. For these reasons, the 
careful selection of cutting parameters is necessary, even indispensable for successful productivity. Increasing productivity with good quality requires Agile methods [7]. The picture carries more information about this topic and its interpretation is simpler [8]. Tribometers designed to respond to the question of development of friction coefficient and wear as function of cutting time, does not propose real results of wear and its shape, particularly during a sudden fracture [9]. Measuring the sizes with better software enables one to have a very high accuracy. These problems which are mainly of tribological nature vary considerably from steels nuance to another. The characterization of materials is essential in developing new steel products [10-12], more efficient and responsive to industrial requirements. The low friction coefficient allows the machining of abrasivematerials such as cast steel, aluminum alloys, tool steels or nickel alloys [13]. Among the many factors influencing the tribological system in metal cutting, the tool performance is mainly affected by temperature and the coefficient of friction $\mu$ (Tool Interface / piece) [14]. Developed to be used at high temperature, superalloys which are based on alloys containing iron, nickel or cobalt have high mechanical properties at high temperatures and low chemical reactivity (inoxidability). The moving fins of the turbine, due to the high mechanical stress to which they are subjected, are almost made of nickel superalloy. Cobalt superalloys are mostly used for fixed blading [15].

The objective of this work is to search the optimized pellets coated with TiAlN, as well as the cutting time. This study has been preceded by a similar research but using pellets coated with TiN. The piece used as a specimen in our tests is a fixed fin of 5001 nuance of a gas turbine subjected to high loads and at a temperature above $1000^{\circ} \mathrm{C}$.

\section{Experimental Procedure}

The tests have been carried out in the numerical control workshop of the MEI company (Sonelgaz) of M'Sila (Algeria) and in mechanical engineering department at the University Med Boudiaf M'Sila.

The purpose of this research is to measure the flank and crater wear at the same cutting inserts in milling operations of a single material with constant cutting parameters, the cutting time is different. The superalloy used in this work is based on cobalt, commercially known as FSX-414 with is characterized with the following mechanical characteristics: an average hardness of $60 \mathrm{HRC}$, a yield strength $0.2 \%$ of 850 $\mathrm{N} / \mathrm{mm} 2$, an ultimate tensile max strength of $1160 \mathrm{~N} / \mathrm{mm} 2$, a Young's modulus of $225 \times 103 \mathrm{~N} / \mathrm{mm} 2$. The chemical composition of this superalloy is given in Table 1 below.

Table 1. Chemical composition of the superalloy FSX-414 (Weight \%, fixed fin 1 Floor 5001)

\begin{tabular}{llllllllllllll}
\hline Co & Ni & Cr & W & Fe & C & Sb & Ag & Mo & Mn & Ti & V & Sn & Nb \\
\hline
\end{tabular} \begin{tabular}{lllllllllllllllllllllll}
\hline 56,89 & 14,68 & 16,8 & 8,03 & 2,52 & 0,25 & 0,01 & 0,05 & 0,35 & 0,3 & 0,04 & 0,05 & 0,01 & 0,056 \\
\hline
\end{tabular}

The platelets used during the machining are made from metal carbide coated with titanium nitride and aluminum (TiAlN). The high performance coating provides a surface hardness which can reach $80 \mathrm{HRC}$. It retains its hardness at high temperatures with the formation of an alumina layer characterized by poor heat conductivity, which allows to evacuate up calories by propagating in chips rather than in the tool itself.

It is possible to reach very high cutting speeds with very little coolant, or even without lubrication, which is the case in the present work.

These tests have been carried out on a 5 axis CNC milling machine (model DMG MORI DMF 180), using a removable cutter surfacing R217 / 220.29 type of round inserts coated with titanium aluminum nitride (TiAlN) by PVD process (RPHT1204M0T-M08 F40M reference).

The cutting conditions are as follows:

Rotation speed $\mathrm{n}=400 \mathrm{r} / \mathrm{min}, \mathrm{Vc}=150 \mathrm{~m} / \mathrm{min}$, feed rate $\mathrm{Fz}=0.075 \mathrm{~mm} /$ tooth;

Cutting depth ap $=0.5 \mathrm{~mm}$.

Inthe first operation, an inclined face surfacing at $45^{\circ}$ is used, followed by a flat surface as shown in figure 1 .

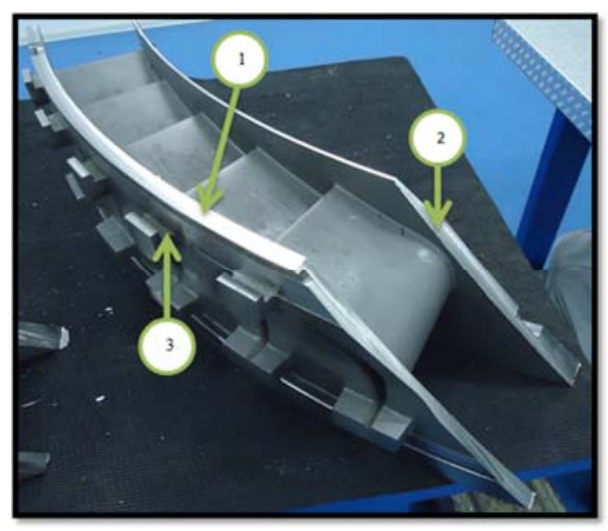

Figure 1. The three surfaces for machining tests on the fixed fin at the first stage fin 5001.

The tests have been conducted with greatest precaution. The general approach of different operations is shown in figure 2 .

Launching of the machining program is as follows:

- Face milling Side 1 (copying tool with round insert of $\Phi 50)$

- face milling of the inclined face 2 (copying tool with round insert $\Phi 50$ );

- face milling of face 3 (planning, grooving and shaping tools $\Phi$ 16).

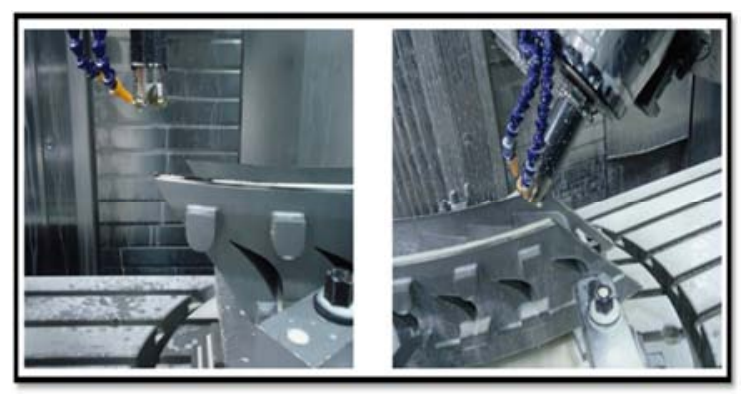

Figure 2. The surfacing operations (side 1 and 2) by copying cutter $\Phi 50$. 
In order to avoid errors on the infeed depth, the diameter before and after machining has been measured according to figure 3:

- Disassembling of the platelets;

- Periodic visual observation of the utilized platelets (cutting face and the flank face);

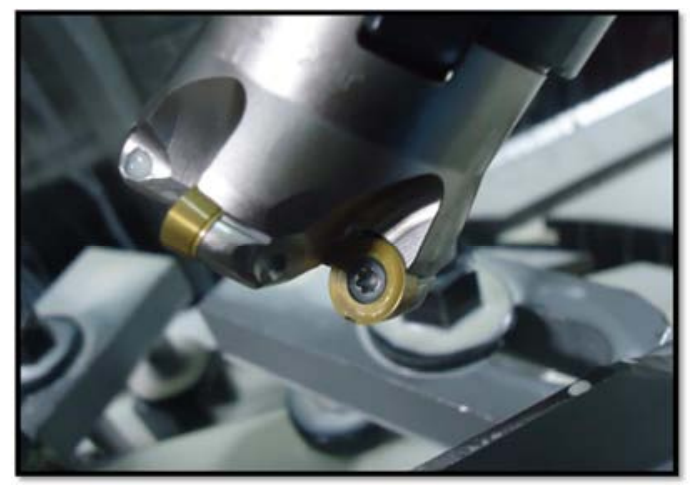

Figure 3. Periodic Visual observation of platelets after the end of each pass.

- Observation and geometric measurement of the different parameters of the platelet (flank wear and crater wear) using a microscope at the end of the last pass;

- Computer processing of data;

- Results analysis.

Both wear criteria adopted in accordance with ISO 3685, whose diagram is shown in figure 4.

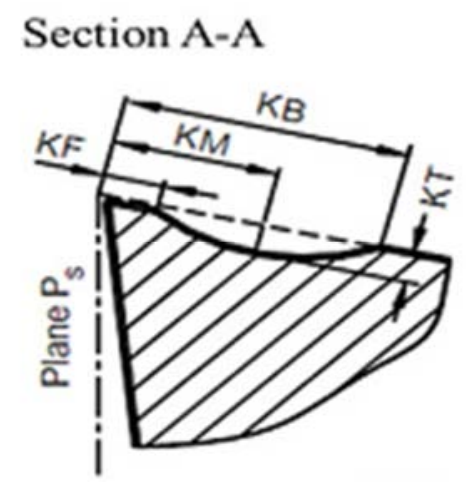

a) $\mathrm{K}_{\mathrm{T}}$ : crater wear;

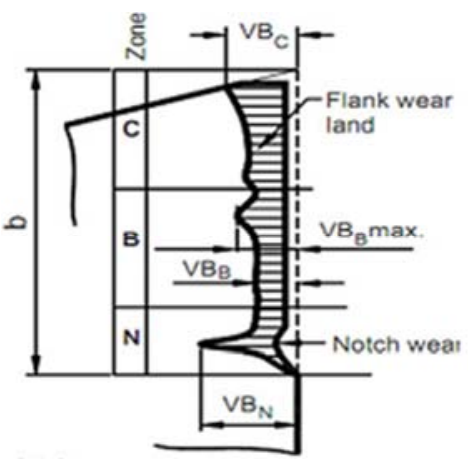

b) $V_{B}$ : flank wear

Figure 4. Diagram of wear criteria used by the ISO 3685 standard.
The insert used in this case has a cutting angle equal to $16^{\circ}$. The cutting position is changed twice on the same insert; one for the first operation and the other for the second operation, this is done on two identical specimens so as to remove the same quantity of material for each machined face.

For the measurement of the wear, a direct technique was adopted, using an optical binocular microscope (Euromex). The zoom magnification is twice the actual size with a magnifying glass (10 times). It is equipped with a Phonotic Touptek camera coupled to a Toupview capture software and image analysis to measure the wear of the cutting edges of the flanks as shown in figure 5.

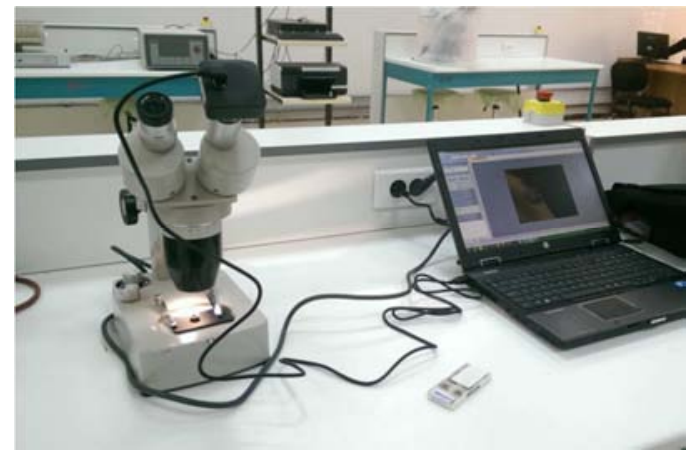

Figure 5. Interface wears measurement (optical binocular microscope, camera and laptop).

A preliminary calculation of the dispersion of the measured values using the following equation (1):

$$
S=\sqrt{\frac{\sum_{i=1}^{n}\left(x_{i}-x_{m}\right)^{2}}{n-1}}
$$

The variation coefficient in \% is: $C V=\frac{S}{x_{m}}$

Where: $\mathrm{x}_{\mathrm{i}}=$ measured values; $\mathrm{x}_{\mathrm{m}}=$ average values; $\mathrm{n}=$ number of measured values.

\section{Results and Discussion}

Figure 6 gives an overview of on the flank wear ofthe platelets coated with TiAlN. The evolution of the wear functions of time:

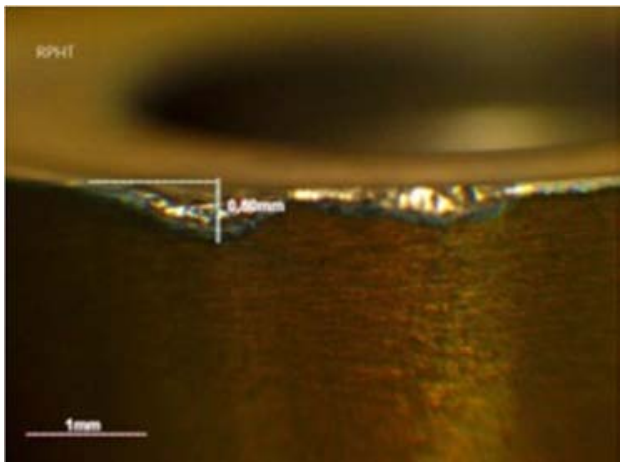

Figure 6(a). $V_{B}=0,50 \mathrm{~mm}$. 


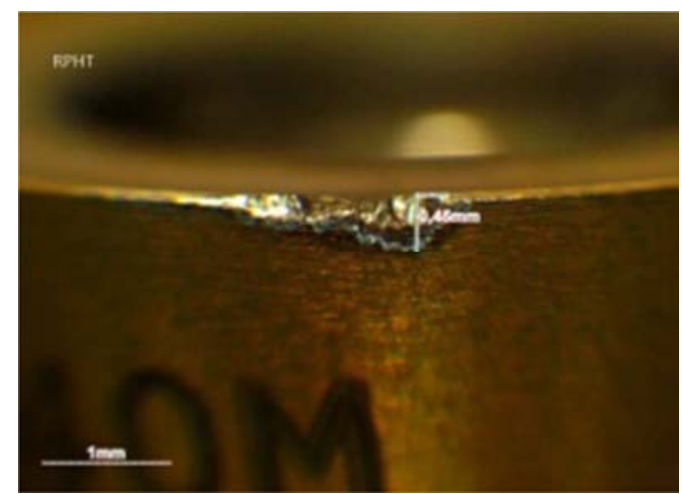

Figure 6(b). $V_{B}=0,45 \mathrm{~mm}$.

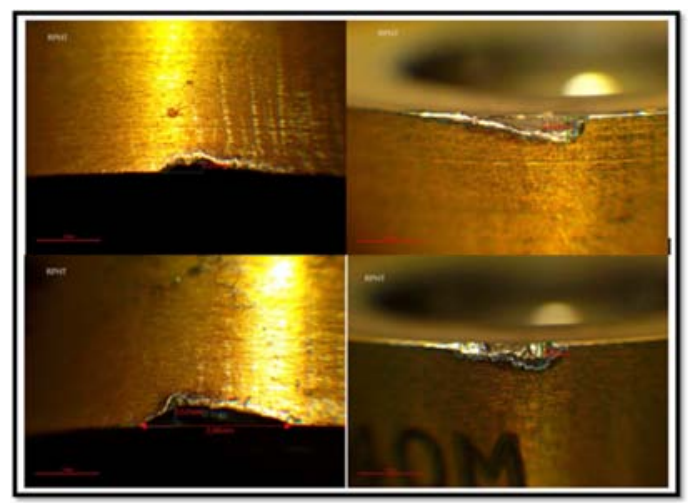

Figure 6(c). Microscopic observation of flank wear on the platelets faces of the type RPHT XOMX coated with TiAlN.

Figure 7 shows the flank wear $\mathrm{V}_{\mathrm{B}}$ of platelets as a function of cutting time. It is noticed that the wear of the inserts coated with TiAlN is more excessive compared to coated inserts with TiN. This is due essentially to the lubrication during machining when using platelets coated with TiN and using dry medium for platelets coated with TiAlN. The two curves show the same aspect (low wear, moderate then accelerated wear). One can notice that the TiN coated platelets resist better to flank wear than TiAlN coated platelets under the same cutting conditions.

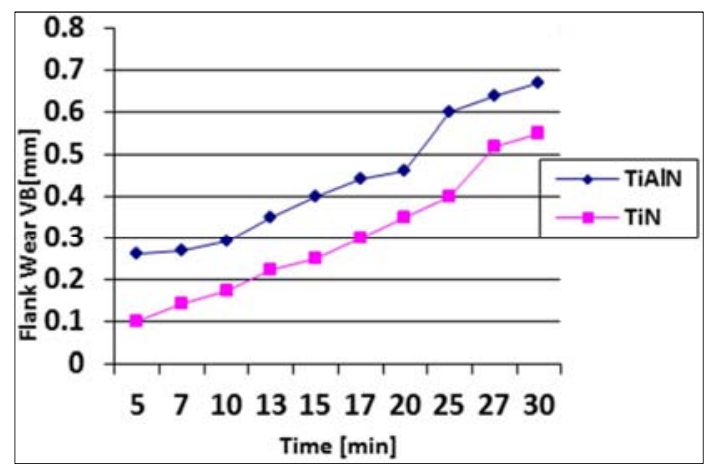

Figure 7. Evolution of the flank wear VB as function of cutting time, obtained with XOMX platelets (F40M type), FSX- 414 piece, $V c=150 \mathrm{~m}$ / $\min , f=0.075(\mathrm{~mm} /$ tooth $)$ and ap $=0.5 \mathrm{~mm}$.

Figure 8 shows the crater wear of the carbide inserts with the two coatings TiN and TiAlN. The crater wear of TiAlN coating is much more pronounced than the TiN coating. The difference is more significant for cutting time beyond 20 minutes.

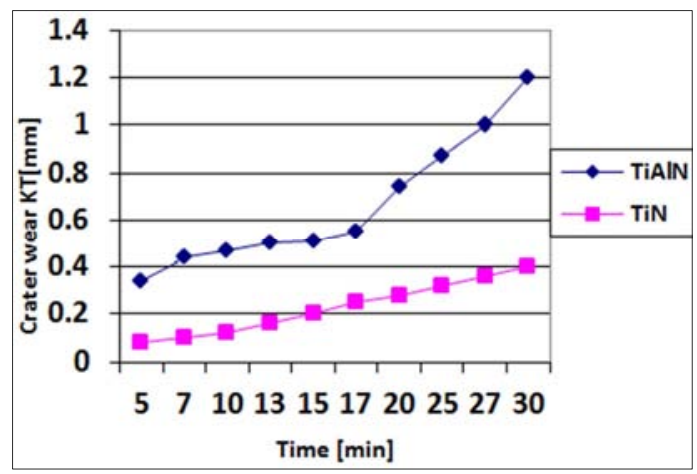

Figure 8. Crater wear Kt evolution as function of cutting time, obtained with RPHT platelets F40M type FSX- 414 piece, $V c=150 \mathrm{~m} / \mathrm{min}, f=0.075(\mathrm{~mm}$ / tooth) and ap $=0.5 \mathrm{~mm}$.

Figures 7 and 8 show the variation of the wear flank $V_{B}$ and crater wear $\mathrm{K}_{\mathrm{T}}$ as function of cutting time.

The insert is under mechanical stress soon as cutting begins and as long as the cut lasts. The importance cutting forces direction cause cracks which are mainly parallel to the cutting edge. Temperature variations occur because the cutting edge is heated during cutting and it cools when it's not heated. The heating and cooling effect causes thermal stresses which cause thermal cracks perpendicular to the cutting edge.

The calculation of the flank average wear measured in this case is given by equation 1 . It is noticed that there is a low dispersion for measured wear for 10 pellets for every test (about $8 \%$ ) due to the high hardness of the material and also due to tribological phenomena generated by the properties which cause a machining difficulty.

For the first test series (figure 7), the differences are not very significant. The flank wear is more remarkable. They sometimes reach the value allowed by ISO $3615 \mathrm{VB}=0.6$ $\mathrm{mm}$ after 27 minutes for TiAlN coatings, and after 32 minutes for TiN coatings. In this case, the tool must be changed; otherwise the surface quality becomes poor.

For the second series of tests 'figure 8), the maximum gap is $0.27 \mathrm{~mm}$ between the two coatings TiN and TiAlN for the same cutting conditions. It is therefore deduced that the TiN coating is better than TiAlN coating. One should note that lubrication is important, in addition and other parameters may be involved in the tribological system.

\section{Conclusion}

The main conclusions which can be drawn from this work are:

The most distinguished wear phenomenon is abrasive wear which occurs by grooving on the flank face of the tool. Abrasion is due to the removal of tool particles caused by harder particles existing in the machined material (see images). Various parameters have an influence on the cutting tools wear as function of time such as the couple tool 
materials / material to be machined. The toughness of this important material needs very specific cutting parameters like reducing the cutting depth and the well defined feed rate ( $\mathrm{Fz}=0.075 \mathrm{~mm} /$ tooth). The crater wear is not very significant in our case, because it is responsible only for the sudden fracture of the beak of the tool. It does not affect the surface quality. Wear is low in the case of TiN coating than in TiAlN coating.

The pellets coated with TiN show better resistance in the case of machining of the superalloy FSX-414.

In fact, the influence of the substrate type on the molecular beam magnetic properties on the epitaxial growth of thin films Fe/Ag has been studied. Therefore, a comparative study between two substrate types: the first type is a substrate of single-crystal $\mathrm{MgO}(001)$, the second is a polycrystalline Silicium ( $\mathrm{Si}$ ) substrate (100) has been carried out.

The flank wear of the main tool mechanism is the phenomenon that occurs during the machining of stainless materials. The flank wear is due to the high abradability of austenite grains, rubbing against the flank face of the tool.

\section{References}

[1] Sebhi. A, Douib. N, Wear Behavior of the Pair Superalloy FXS-414 and Cutting Tool-Carbide Coated TiN. Journal of Machinery Manufacturing and Automation., Vol. 5 Iss. 1, 2016, PP. 1-7.

[2] Sebhi. A, Osmani. H, Rech. Joel, Tribological Behaviour of Coated Carbide Tools during Turning of Steels with Improved Machinability, Strojniški vestnik - Journal of Mechanical Engineering 58(2012)12, DOI:10.5545/sv-jme, 2012.561, PP 744-749.

[3] Rech. J, Influence of cutting tool coatings on the tribological phenomena at the tool-chip interface in orthogonal dry cutting. Surf. Coat, Technol., 200, 2006, PP 5132-5139.

[4] Grzesik. W, Zalisz. Z, and Nieslony. P, Friction and wear testing of multilayer coatings on carbide substrates for dry machining applications, Surf. Coat. Technol, 155, 2002, PP $37-45$.
[5] Zemzemi. F, Rech. J, Ben Salem. W, Kapsa. P, and Dogui. A, Development of a friction model for the tool-chip-workpiece, 2008.

[6] Bouchelaghem. H, Yallesse. M. A, Amirat. A., Belhadi. S, Wear behaviour of CBN tool when turning hardened AISI D3; steel, Mechanika, vol. 65 , no. 3, 2007, pp. 57-65.

[7] Gabriela Robiolo, Daniel Grane, Do Agile Methods Increase Productivity and Quality International Journal of Mechanical Engineering and Applications, Science PG, Published Online: Apr. 20, 2014, DOI: 10.11648/j.ajsea.20140301.11, Pages: 111.

[8] Qi. H. S, Mills. B, On the formation mechanism of adherent layers on a cutting tool, Wear, vol. 198, no. 1-2, p. 192-196, DOI:10.1016/0043-1648(96)80023-8, 1996.

[9] Farhat. Z. N, Wear mechanism of CBN cutting tool during high-speed machining of mold steel, Materials Science and Engineering, vol. 361, no. 1-2, pp. 100-110, DOI:10.1016/S0921-5093(03)00503-3, (2003).

[10] J. kopac, M. sokovic, S. dolinsek, Tribology of coated tools in conventional ahd HSC machining. J. of materials processin Technology; 118,377-384, 2001.

[11] Sandvik Coromant siteweb, Wear of the milling tool (Expertise), http:/www.sandvi.coromant.com/.

[12] A. Hebbar. D. Kaidameur, D. Ouinas, Modelling of the wear of song tooling materials, International review of mechanical engineering (IREME), Vol. 1, January 2007, pp 86-91.

[13] S. Y. Hong, I. Markus, and W.-C. Jiong, New cooling approach and tool life improvement in cryogenitic machining of titanium alloy Ti-6Al-4V, International of machine tools and Manufacture, vol.41, no, 15, 2001, PP. 2245-2260.

[14] Andinet Kumella Eticha, Analysis of the Performance of Disc Brake System of Addis Ababa Light Rail Transit Using Temperature and Coefficient of Friction as a Parameter, International Journal of Mechanical Engineering and Applications, Science PG, Published Online: Nov. 16, 2016, Pages: 205-211.

[15] Matériaux de turbines à combustion - Evolution des tendances (Direction des études et recherches) EDF Electricité de France Octobre 1992. 